\title{
FAK competes for Src to promote migration against invasion in melanoma cells
}

\author{
K Kolli-Bouhafs ${ }^{1,2}$, E Sick ${ }^{1,2}$, F Noulet ${ }^{1,2}$, J-P Gies ${ }^{1,2}$, J De Mey ${ }^{1,2}$ and P Rondé ${ }^{*, 1,2}$
}

Melanoma is one of the most deadly cancers because of its high propensity to metastasis, a process that requires migration and invasion of tumor cells driven by the regulated formation of adhesives structures like focal adhesions (FAs) and invasive structures like invadopodia. FAK, the major kinase of FAs, has been implicated in many cellular processes, including migration and invasion. In this study, we investigated the role of FAK in the regulation of invasion. We report that suppression of FAK in B16F10 melanoma cells led to increased invadopodia formation and invasion through Matrigel, but impaired migration. These effects are rescued by FAK WT but not by FAK $^{\mathrm{Y} 397 \mathrm{~F}}$ reexpression. Invadopodia formation requires local Src activation downstream of FAK and in a FAK phosphorylation-dependant manner. FAK deletion correlates with increased phosphorylation of Tks-5 (tyrosine kinase substrate with five SH3 domain) and reactive oxygen species production. In conclusion, our data show that FAK is able to mediate opposite effects on cell migration and invasion. Accordingly, beneficial effects of FAK inhibition are context dependent and may depend on the cell response to environmental cues and/or on the primary or secondary changes that melanoma experienced through the invasion cycle.

Cell Death and Disease (2014) 5, e1379; doi:10.1038/cddis.2014.329; published online 14 August 2014

Patients with spreading melanoma diseases have a very poor prognosis with a 5 -year survival rate $<5 \%$. The metastatic spread of melanoma is a complex process involving several genetic alterations. In melanoma, ${ }^{1}$ as in many highly invasive cancer cell types like head and neck squamous cell carcinoma ${ }^{2}$ or breast carcinoma, ${ }^{3}$ specialized matrix-degrading organelles termed invadopodia have been identified. Invadopodia consist of dynamic actin-based protrusions of 0,1 to $2 \mu \mathrm{m}$ in diameter emanating from the ventral edge of tumor cells. ${ }^{4}$ Besides their actin scaffold, these structures are enriched in proteolytic enzymes such as matrix metalloproteinases (MMPs), which mediate extracellular matrix (ECM) degradation. Indeed, MMP are upregulated in invasive melanoma and there is extensive evidence that they have a role in promoting the dissemination of melanoma. ${ }^{5-7}$ Several proteins like integrins, Src and paxillin, found at sites of cell adhesion to the matrix, are also present in invadopodia. ${ }^{8,9} \mathrm{On}$ the other hand, other proteins like the Src substrate proteins cortactin $^{10}$ and the tyrosine kinase substrate with five $\mathrm{SH} 3$ domain $(T k s-5)^{11}$ are specifically localized at invadopodia and not found at focal adhesion (FA). In addition, reactive oxygen species (ROS) ${ }^{12}$ have been localized at invadopodia and are supposed to have a prominent role in inducing invadopodia function. ${ }^{13,14}$ Although significant efforts have been made to characterize components of invadopodia, the precise mechanisms of their regulation, especially in a melanoma context, remain poorly understood.

Tumor invasion is a multistep process that requires cell adhesion to the environing substratum, migration and invasion. In many cell types, migration requires fine control of FA turn-over. FAs are formed by the cluster of up to 200 proteins ${ }^{15}$ ensuring cell anchorage to the ECM. The cyclic process of FA formation and disruption is crucial for cell migration. Because both anchorage and migration involve cellular interactions with ECM components, FAs are endowed with transmembrane ECM receptor proteins such as integrins that interact with ECM molecules and intra-cellular proteins composed of scaffold proteins, as well as signal-transducing molecules. Among those, focal adhesion kinase (FAK) is a crucial signaling protein that integrates signals from integrins to the actin filaments during cell migration. ${ }^{16}$ Structurally, FAK is a $125-\mathrm{kDa}$ protein that contains an $\mathrm{N}$-terminal 4.1 -ezrinradixin-moesin domain, a central kinase domain and a C-terminal domain that contains the focal adhesion targeting site. ${ }^{17}$ The phosphorylation of FAK at Y397 creates a binding site for Src, which can phosphorylate other tyrosines on the FAK sequence, thus creating new binding sites for $\mathrm{SH} 2$ domain-containing proteins.

FAK is involved in many aspects of the metastatic process and thus, overexpression, hyperphosphorylation and/or elevated activity of FAK have been reported in a variety of human cancers, including sarcomas and carcinomas of the breast, colon, thyroid, prostate, oral cavity, liver, stomach and ovary. ${ }^{18}$ In human melanoma cell lines, early studies reported high FAK expression and requirement of FAK for cell substrate adhesion. ${ }^{19}$ Later, it was reported that FAK promotes the aggressive melanoma phenotype. ${ }^{20}$ Indeed, immunohistochemical analyses revealed high levels of FAK

\footnotetext{
${ }^{1}$ CNRS, UMR 7213, Laboratoire de Biophotonique et Pharmacologie, Illkirch, France and ${ }^{2}$ Université de Strasbourg, Faculté de Pharmacie, Illkirch, France *Corresponding author: P Rondé, CNRS, UMR 7213, Faculté de Pharmacie, Université de Strasbourg, 74 rte du Rhin, 67401 Illkirch, France. Tel: + 33368854184 ; Fax: + 333688543 13; E-mail: philippe.ronde@ unistra.fr

Abbreviations: MMP, matrix metalloproteinases; FA, focal adhesion; FAK, focal adhesion kinase; ECM, extracellular matrix; Tks-5, tyrosine kinase substrate with five SH3 domain; mda-9, melanoma differentiation-associated gene-9; PKC $\alpha$, protein kinase $\mathrm{C} \alpha$; ROS, reactive oxygen species; DAPI, 4',6-diamidino-2-phenylindole; FRAP, fluorescence recovery after photobleaching; TIRF, total internal reflection fluorescence

Received 06.2.14; revised 16.6.14; accepted 01.7.14; Edited by Z-X Xiao
} 
phosphorylation at Tyr397 and Tyr576, a marker of FAK kinase activity, in late-stage cutaneous and uveal melanoma, which correlated with their increased invasion and migration properties. ${ }^{21}$ Furthermore, melanoma differentiation-associated gene-9 (mda-9)/syntenin was also reported to mediate adhesion-dependant activation of protein kinase $\mathrm{C} \alpha(\mathrm{PKC} \alpha)$ and FAK in melanoma cells. Thus, inhibiting either mda-9/ syntenin or $\mathrm{PKC} \alpha$ suppressed fibronectin-induced formation of integrin- $\beta 1 / F A K / c-S r c$ signaling complexes and reduced migration and invasion toward fibronectin. ${ }^{22}$ Therefore, FAK appears to be a major player of melanoma invasion, but how this kinase controls the formation and proteolytic activity of invadopodia in melanoma cells was never investigated.

In this study, we uncovered a surprising negative regulation of invadopodia activity in B16F10 cells by FAK. The depletion of FAK was associated with increased ROS production and Tks-5 phosphorylation. Using mutation of FAK at Tyr397, a binding site for Src, we found that these sites are implicated in FAK-mediated inhibition of invadopodia activity. In addition, we report that this mutation induced decreased migration speed but increased invasive properties. Taken together, our data suggest a competition between FA and invadopodia substrates for Src phosphorylation that might depend on environmental cues, thus leading to the engagement of either migration or degradation pathways.

\section{Results}

FAK deletion is associated with increased proteloytic activity of invadopodia. FAK has long been known to promote migration and invasion of many cellular cancer types, among which is the murine melanoma B16F10 cell line. ${ }^{23}$ However, whether and how FAK affects invadopodia activity especially in the melanoma context remains poorly documented. To begin investigating the role of FAK in melanoma B16F10 invasion, we compared the ability of invadopodia to degrade the ECM in control and FAKdeficient cells. For this purpose, we used siRNA directed against the $5^{\prime}-U T R$ region of FAK to deplete cells from endogenous FAK. Western blot analysis of FAK expression revealed that knockdown efficiency reached 85.33 and $84.24 \%$ after 48 and $72 \mathrm{~h}$ of siRNA transfection, respectively (Figure 1a). Invadopodia activity was quantified by their ability to degrade the ECM using Alexa-405 gelatin as a substrate. As shown in Figure 1b, B16F10 cells can spontaneously form invadopodia after $16 \mathrm{~h}$ of culture on fluorescent gelatin. Surprisingly, quantification of fluorescent gelatin degradation revealed that matrix degradation was 14 times higher in FAK-deficient cells as compared with control cells (Figure 1b).

Src is necessary for the degradation activity of invadopodia downstream of FAK. To investigate whether Src, a binding partner of FAK, is implicated in the process of matrix degradation, control and FAK-deficient B16F10 cells were treated with PP2, an inhibitor of Src kinase. We find that PP2 treatment completely abolished active invadopodia in control (data not shown) and FAK-deficient B16F10 cells (Figure 2a), thus establishing the essential role of Src in the control of matrix degradation. It should also be noted that, although Src a
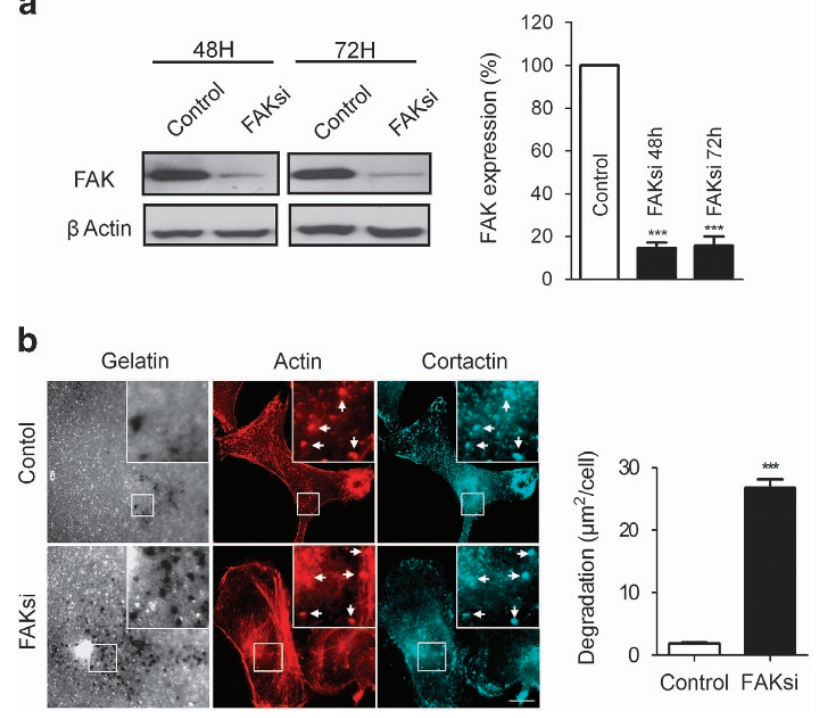

Figure 1 Depletion of FAK in B16F10 cells increases invadopodia activity. (a) B16F10 cells were transiently transfected with either control siRNA or FAK siRNA. Cellular extracts collected at 48 and $72 \mathrm{~h}$ were analyzed by western blotting and probed for FAK and actin as a loading control. Inhibition of FAK expression to $85 \%$ of control was efficient up to $72 \mathrm{~h}$ after transfection. (b) B16F10 cells transiently transfected with control siRNA and FAK siRNA were cultured on Alexa fluor 405 gelatin coverslips (gray) and labeled for actin (red) and cortactin (cyan). Insets show magnified views of invadopodia. Note the colocalization of actin and cortactin at dotlike invadopodia (arrows). Scale bar: $20 \mu \mathrm{m}$. Quantification of degradation areas shows increased invadopodia activity in cells depleted for FAK as compared with control cells' transfection $\left(n=3,{ }^{\star \star \star} P<0.0001\right)$

inhibition blocked invadopodia activity, further FAK depletion was unable to restore matrix degradation, thus suggesting that Src acts downstream of FAK in the control of invadopodia activity. The question then arises as to whether the increased matrix degradation observed in FAK-deficient B16F10 cells could be due to an increase in Src activity. For this purpose, western blot analysis using a phosphospecific Ab directed against Src-Tyr416, which constitutes a marker of Src activation, ${ }^{12,24}$ was performed. In an unexpected way, no variation in Src phosphorylation at Tyr416 was observed between control and FAK-deficient B16F10 cells (Figure 2b). This result suggests that FAK does not alter Src activity in melanoma cells. Therefore, as Src is involved in invadopodia activity, FAK may affect downstream substrates of the Src kinase implicated in invadopodia formation and/or the turnover of Src at specific cellular locations.

Next we evaluated whether the phosphorylation state of relevant Src substrates has been altered in FAK-deficient cells. At invadopodia, several Src substrates are phosphorylated in a way critical for their functional activity, including cortactin $^{25}$ and Tks-5. ${ }^{26}$ Tks-5 appears localized at invadopodia in cancer cells and has a prominent role in their formation. ${ }^{27}$ By western blotting, we show that Tks-5 phosphorylation was increased in FAK-deficient cells as compared with control (Figure 3a). Co-immunoprecipitation experiments confirm the global increase of tyrosine phosphorylation of Tks-5 but not cortactin in FAK-deficient B16F10 cells. Moreover, overexpression of active Src or active SrcmCherry greatly enhances Tks-5 phosphorylation level as 
a

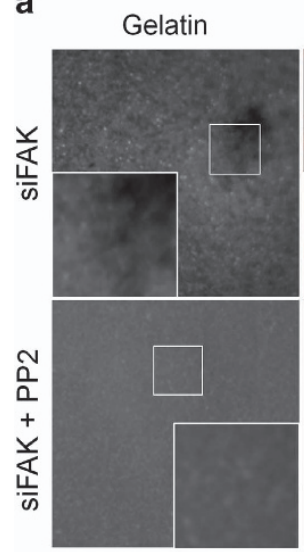

Actin

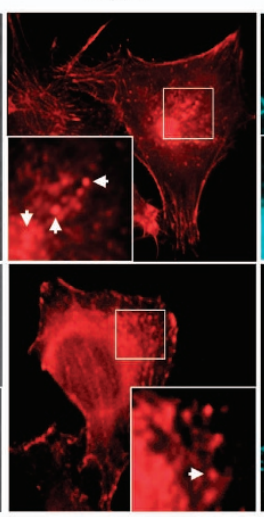

b

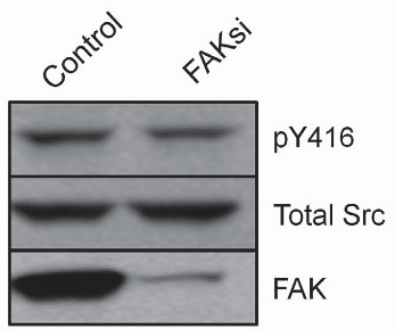

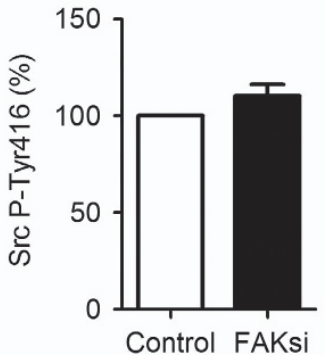

Figure 2 Src activity is necessary for invadopodia formation. (a) B16F10 cells transiently transfected with control siRNA or FAK siRNA were cultured on Alexa fluor 405 gelatin coverslips and treated or not with the Src inhibitor PP2. No degradation was observed in both control (data not shown) and cells treated with FAK siRNA after Src inhibition. Insets show magnified views of invadopodia. Arrows indicate the colocalization of actin and cortactin at dot-like invadopodia. Scale bar: $20 \mu \mathrm{m}$. (b) Cellular extracts were analyzed by western blotting and probed for phosphor-Tyr416-Src. The Graph shows no increase in Src activity after FAK depletion

revealed by an immunoprecipitation experiment using a Tks- 5 antibody (Figure 3b, left; Supplementary Figure 1). This suggests that the formation of the FAK-Src complex hinders the phosphorylation of Tks-5, although once the FAK-Src complex is disrupted due to FAK depletion, Src acquires the ability to phosphorylate Tks-5. To investigate whether Src is indeed a limiting factor in the phosphorylation of Tks-5, B16F10 cells were transfected with the activated form of Src, SrcY530F. As shown in Figure 3b, expression of the activated form of Src coupled to mCherry did not change the basal level of Tks-5 in both control and FAK-deficient cells. On the other hand, immunoprecipitation studies revealed an increase in the phosphorylation state of Tks-5 in B16F10 cells transfected with activated $\mathrm{Src}$ as expected for a Src substrate. This increase in Tks-5 phosphorylation is not further augmented in FAK-deficient cells, suggesting that, in the absence of FAK, Src-mediated Tks-5 phosphorylation already reached a plateau. This suggests a competitive role of FAK and Tks-5 for Src phosphorylation. To address this question, we examined the cellular localization of active $\mathrm{Src}$ in control and FAK-deficient cells. In control cells, we detected robust staining of phospho-Src-Tyr416 at FAs identified by talin labeling (Figure 4a, top) and at cytoplasmic dots, some of

a

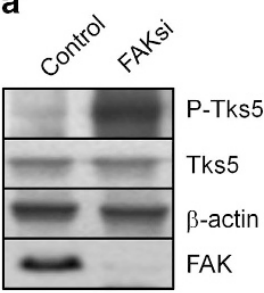

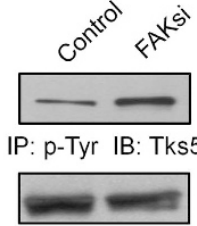

IB: Tks5

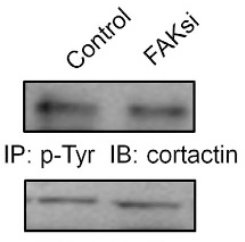

IB: cortactin b
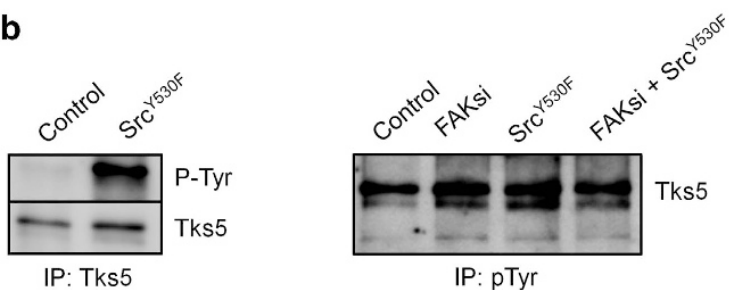

IP: pTyr
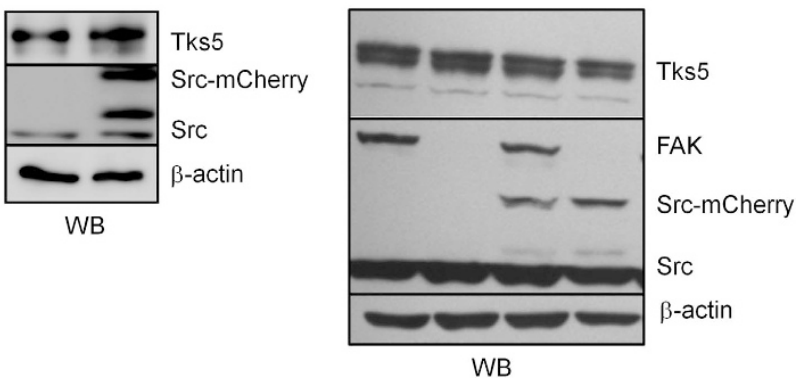

Figure 3 FAK modulates Tks-5 phosphorylation upstream of Src. (a) Cell extracts from B16F10 cells transiently transfected with control siRNA or FAK siRNA were analyzed by western blotting (WB) and immunoprecipitation (IP) and probed for phosphotyrosines, Tks-5, actin, cortactin and FAK. Note the increase in Tks-5 phosphorylation state on FAK deletion. (b) Representative western blot showing B16F10 cells transiently transfected with $\mathrm{Src}^{\mathrm{Y} 530 \mathrm{~F}}$-mCherry, immunoprecipitated using anti-Tks-5 Ab and blotted for phosphotyrosines and Tks-5. The expression level of proteins in the corresponding cell lysates is shown. Note the increase in Tks-5 phosphorylation state on Src ${ }^{\mathrm{Y} 530 \mathrm{~F}}$ expression (left panel). Representative western blots showing B16F10 cells transiently transfected with control siRNA or FAK siRNA and Src ${ }^{\mathrm{Y} 30 \mathrm{~F}}$-mCherry immunoprecipitated using anti-phospho-Tyr Ab and blotted for Tks-5. Note the equal increase in the phosphorylation state of Tks-5 in FAK-depleted cells with or without $\mathrm{SrC}^{\mathrm{Y} 530 \mathrm{~F}}$ expression. The expression level of proteins in the corresponding cell lysate is shown (right panel)

which being invadopodia as revealed by actin/cortactin staining (Figure 4b, top). On the contrary, in FAKsi-treated cells, active Src is no longer localized at FAs (Figure 4a, bottom) but displays increased localization at invadopodia (Figure 4b, bottom). Together with the established role of Src in Tks-5 phosphorylation previously demonstrated, this is consistent with a role of FAK in controlling the localization of active Src, thus leading to the differential regulation of FA and invadopodia activities.

ROS are necessary for the degradative activity of invadopodia. Recently, a correlation has been established between ROS production, Tks- 5 expression and the formation of invadopodia. ${ }^{13}$ As we observed an increase in the phosphorylation state of Tks-5 in our cells, we measured the level of ROS in control and FAK-deficient B16F10 cells. As expected, we found a $38 \%$ increase in ROS production on FAK depletion (Figure 5a). Moreover, cells' treatment with the ROS inhibitor MnTMpyP blocked the degradative activity 
a
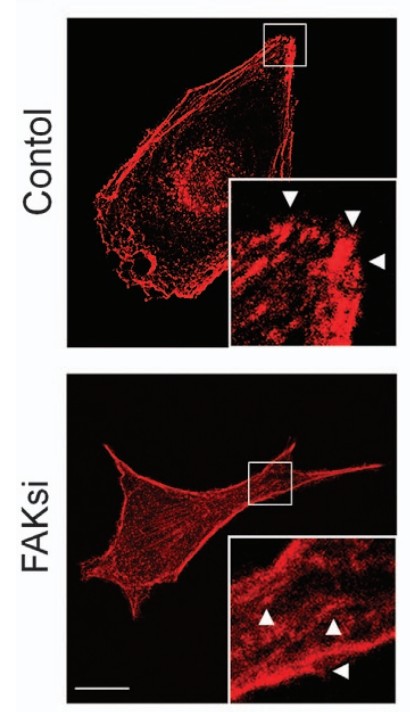

b
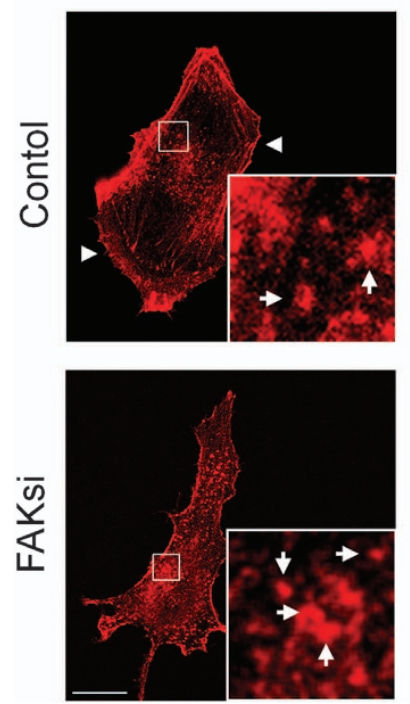

Talin
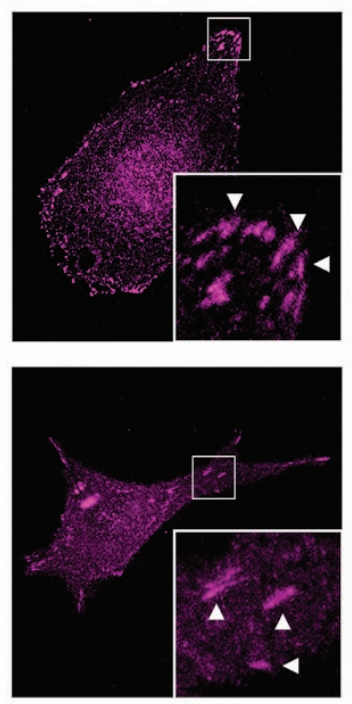

Cortactin
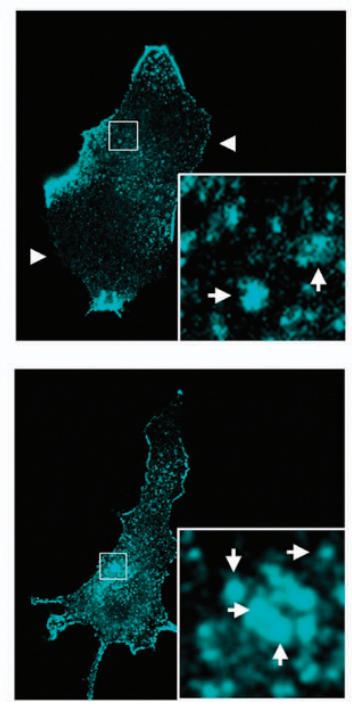

P-Src
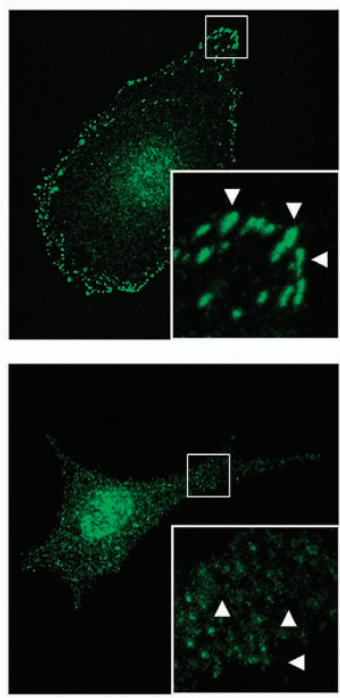

P-Src
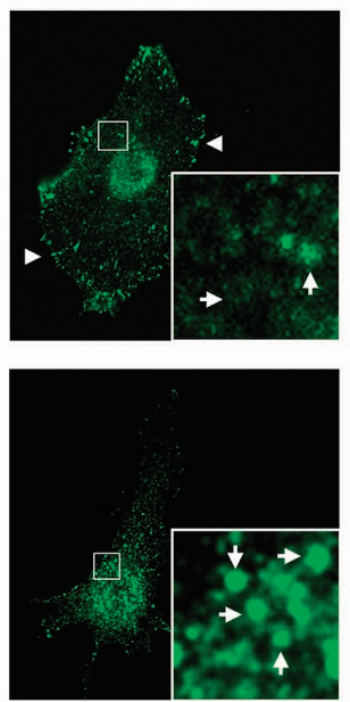

Superposition
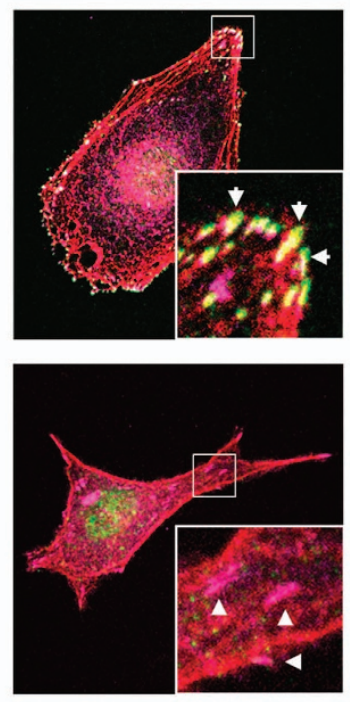

Superposition
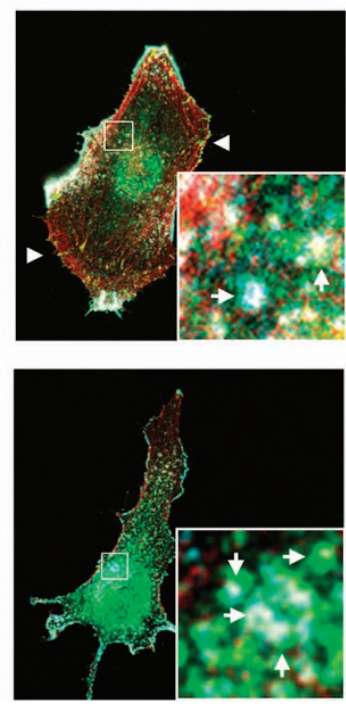

Figure 4 FAK is required for localization of active Src at FA but not at invadopodia. (a) Confocal images of fixed control or FAK siRNA-treated cells immunostained for actin (left panel), talin (middle panel) or phosphorylated Src at Tyr416 (right panel). Note the lack of localization of active Src at FAs (arrowheads) identified by talin staining in FAK siRNA-treated cells as compared with control cells. (b) Confocal images of fixed control or FAK siRNA-treated cells immunostained for actin (left panel), cortactin (middle panel) or phosphorylated Src (right panel). Note the increased level of active Src at dot-like invadopodia (arrows) identified by actin/cortactin staining in FAK-deficient cells as compared with control cells. Scale bars: $20 \mu \mathrm{m}$

of invadopodia in both control and FAK-deficient B16F10 cells, thus confirming the essential role of ROS production in the control of invadopodia activity (Figure $5 \mathrm{~b}$ ). These results suggest that FAK, via its interaction with Src, downregulates Tks-5 phosphorylation, thus altering ROS production.

FAK phosphorylation at Tyr397 negatively regulates invadopodia activity. Next we investigated which molecular determinants are responsible for FAK-mediated decreased invadopodia activity in B16F10 cells. For this purpose, we first explored the role of the FAK-Tyr397 phosphorylation site, as this site has been previously shown to be a binding site for the $\mathrm{SH} 2$ domain of Src. Thus, B16F10 cells expressing FAK WT or FAK-Y397F coupled to GFP derivatives were generated and transfected with siRNA directed against the $5^{\prime}$-UTR region of FAK. As this region is exclusively found in endogenous FAK but not in either FAK WT or FAK-Y397F constructs, targeting the $5^{\prime}$-UTR region allows an almost complete extinction of endogenous FAK without affecting the expression of exogenous FAK forms as demonstrated in Figure 6a. The subcellular localization of the FAK forms revealed that, although both FAK-GFP and FAKY397F-GFP were correctly addressed at FAs, none of the constructs were found at invadopodia as demonstrated by the lack of colocalization of FAK with actin and cortactin (Figure 6b) known to be co-enriched at invadopodia. ${ }^{3}$ 

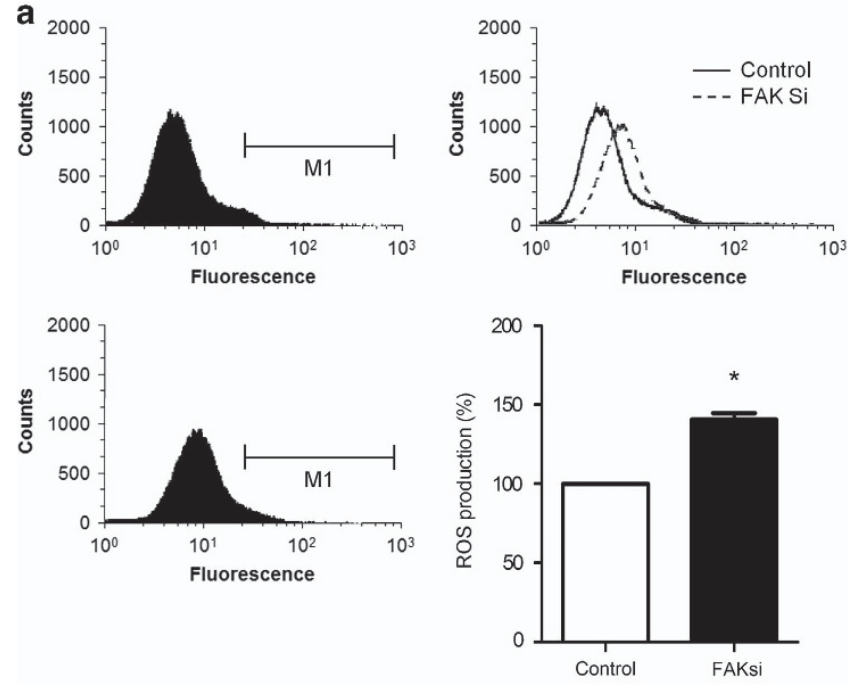

b

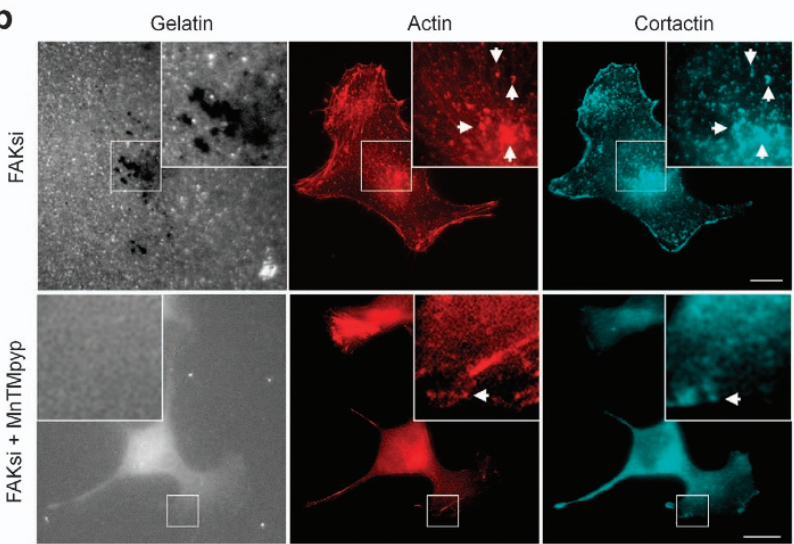

Figure 5 ROS are necessary for invadopodia activity, and are increased in FAK-depleted cells. (a) B16F10 cells transiently transfected with control siRNA or FAK siRNA were treated with the ROS reporter CM-H2DCF-DA for 20 min and then, ROS production was analyzed by flow cytometry. Representative histograms are shown. Fluorescence intensity is plotted versus the number of cells. Quantification of ROS production shows a $38 \%$ increase in fluorescence in cells depleted for FAK $\left(n=4,{ }^{*} P<0.001\right)$. (b) B16F10 cells transiently transfected with control siRNA and FAK siRNA were cultured on Alexa fluor 405 gelatin coverslips (gray) in the presence or not of the ROS inhibitor MnTMpyP, and then labeled for actin (red) and cortactin (cyan). Representative image shows complete inhibition of invadopodia activity in the presence of MnTMpyP. Insets show magnified views of invadopodia. Arrows indicate the colocalization of actin and cortactin at dot-like invadopodia. Scale bar: $20 \mu \mathrm{m}$

To examine the effect of FAK and FAK mutant reexpression in a FAK-null context, we quantified the number of invadopodia in each context. Our results show that cells reexpressing FAK WT but not FAK-Y397F displayed significantly less invadopodia as compared with FAKdeficient cells, thus suggesting that FAK phosphorylation at Tyr397 in FAs is necessary for FAK-mediated inhibition of invadopodia formation. As FAK promotes a reduction in the number of invadopodia displayed per cells, we analyzed the degradative activity of FAK-null cells after reexpression of FAK WT or FAK-Y397F. As shown in Figure 7a, the expression of FAK-Y397F but not FAK WT increased the degradative activity of invadopodia reaching $24.10 \mu \mathrm{m}^{2} /$ cell, close to the value obtained with FAK depletion $\left(24.90 \mu \mathrm{m}^{2} /\right.$ cell). This shows that the loss of phosphorylation at Tyr397 has a similar effect on the degradative activity of invadopodia as FAK depletion, pinpointing that the phosphorylation at Tyr397 is necessary for FAK to negatively regulate invadopodia function. Moreover, this FAK mutant also triggered an increase in Tks-5 phosphorylation (Figure 7b) and ROS production (Figure 7c), suggesting that the disruption of FAK-Src interaction had enabled Src to promote invadopodia activity by phosphorylating Tks-5 and subsequent ROS production.

FAK deletion reduces cell migration but increases cell invasion. We next investigated whether FAK deletion impaired cell migration. The migration properties of control and FAK siRNA-treated cells reexpressing wild-type FAK or FAK-Y397F cells were evaluated in the Boyden chamber assay with $10 \%$ FBS used as a chemoattractant. This showed that the migration speed of FAK siRNA-treated cells was decreased by $58.4 \%$ as compared with non-treated cells. This effect was abolished by reexpression of wild-type FAK but not that of FAK-Y397F (Figure 8a). Thus, as already demonstrated in various other cancer and non-cancer cell lines, FAK expression is necessary for melanoma cell migration. As FAK deletion increases invadopodia activity but decreases cell migration, the invasive properties of B16F10 melanoma cells were evaluated in invasion assays in which the cells degrade and migrate through Matrigel. To evaluate the invasive properties, without accounting for the intrinsic migration properties, we calculated the invasion index by dividing the percentage of cells invading through Matrigel-coated inserts with the percentage of cells migrating through non-coated insert. This showed that FAK-deficient cells were significantly more invasive than control cells. This effect was abolished by reexpression of wild-type FAK but not that of FAK-Y397F (Figure 8b).

FAK is phosphorylated and highly dynamic at FAs. As FAK is not localized at invadopodia but phosphorylated FAK alters invadopodia activity, we analyzed the localization of FAK phosphorylated at Tyr397 in B16F10 cells. Using a phosphospecific Ab directed against FAK-Tyr397, we found that FAK is mainly phosphorylated at FAs with no specific staining at invadopodia. Therefore, FAK residency time at FAs will determine its phosphorylation state and thus the degree of Src accumulation at FAs. To analyze the dynamics of FAK at FA, the mobility of FAK was assessed by fluorescence recovery after photobleaching (FRAP) experiments. To this end, the GFP moiety contained in the FAK fusion protein present in FAs randomly selected at the periphery of the cells was photobleached with short highpower 491-nm excitation using a Cobolt Calypso Laser, and the recovery of fluorescence in the bleached regions was followed by time-lapse imaging over the ensuing $100 \mathrm{~s}$ (Figure 9). Because adhesions are localized at thin cell edges near the interface with the glass, high signal/noise ratio could be achieved using total internal reflection fluorescence (TIRF) microscopy for time-lapse imaging. Using this method, we found unexpectedly fast dynamics of FAK at Fas, yielding a $t_{1 / 2}$ of $7.53 \pm 1.7 \mathrm{~s}$ together with a high mobile fraction of $92.03 \%$. 
a

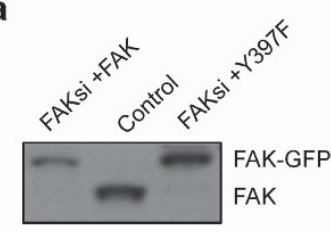

b

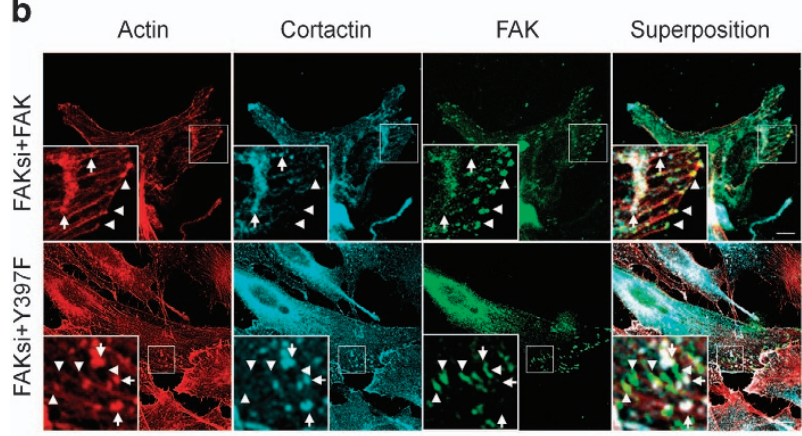

C
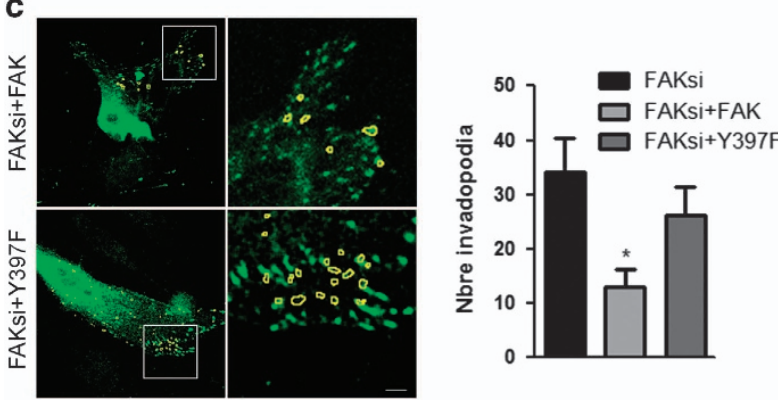

Figure 6 FAK mutation at Tyr397 increases invadopodia formation. (a) B16F10 cells were transiently transfected with either control siRNA or FAK siRNA and FAKGFP or FAK ${ }^{\mathrm{Y} 397 \mathrm{~F}}$-GFP. Representative western blots showing FAK expression in the different conditions. Note the reexpression of FAK in siRNA-treated cells transfected with FAK WT or FAK ${ }^{\text {Y397F }}$. (b) Confocal images of fixed FAK siRNAtreated cells expressing wild-type or mutant FAK-GFP (middle right panel) and immunostained for actin (left panel) or cortactin (middle left panel). Note the localization of FAK at FAs (arrowheads) and the absence of FAK at invadopodia (arrows) identified by actin/cortactin staining in cortical dot-like structures. Scale bars: $5 \mu \mathrm{m}$. (c) Analysis of the number of invadopodia per cell in the different conditions above. Invadopodia segmented using both actin and cortactin staining appear in yellow on the FAK image (right). Quantification reveals a significant reduction in the number of invadopodia in FAK siRNA-treated cells transfected with FAK WT but not FAK ${ }^{\mathrm{Y} 397 \mathrm{~F}}$ ( ${ }^{*} P<0.02, n=5$ with $65-170$ invadopodia analyzed per conditions)

\section{Discussion}

Despite recent progress in our knowledge of the molecular mechanisms implicated in melanoma dissemination, metastatic melanoma still accounts for more than $80 \%$ of deaths by skin cancer. This is mainly due to its high plasticity and high resistance to existing therapies. ${ }^{28,29}$ Therefore, identification of new therapeutic targets is urgently needed. Today, invadopodia are thought to promote the invasive properties of cancer cells by clustering proteases involved in ECM degradation. ${ }^{30,31}$ Recently, these structures have been identified in many metastasic melanoma from both human and mouse origin. ${ }^{31-33}$ Although significant advances have been made in understanding the role of FAK in migration processes, ${ }^{34}$ its role in the regulation of invadopodia dynamics

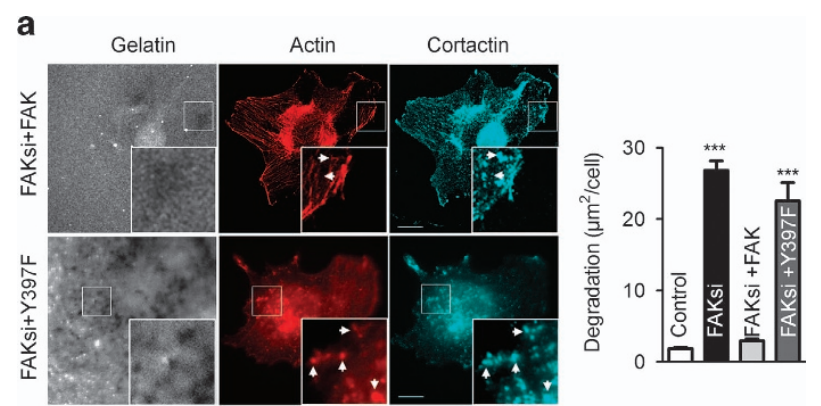

b
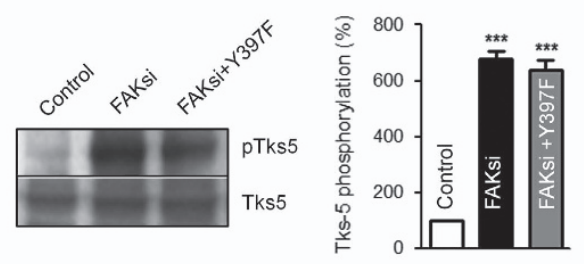

C

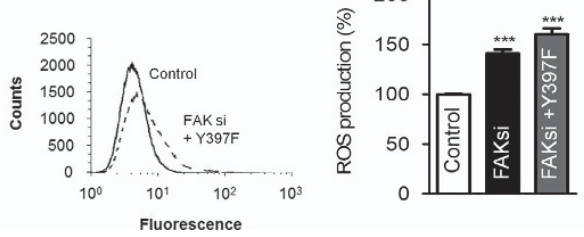

Figure 7 FAK mutation on Tyr397 increases invadopodia activity, ROS production and Tks-5 phosphorylation. (a) B16F10 cells transiently transfected with FAK siRNA and FAK WT or FAK ${ }^{Y 397 F}$ were cultured on Alexa fluor 405 gelatin coverslips (gray) and labeled for actin (red) and cortactin (cyan). Scale bars: $20 \mu \mathrm{m}$. Quantification of degradation areas shows increased invadopodia activity in cells depleted for FAK, which is overcome by FAK WT but not FAK ${ }^{\mathrm{Y} 397 \mathrm{~F}}$ reexpression $\left(n=3,{ }^{* * *} P<0.0001\right)$. (b) Extracts from B16F10 cells transiently transfected with FAK siRNA and FAK ${ }^{\text {Y397F }}$ were analyzed by immunoblotting and probed for Tks-5 and phosphotyrosines. Note the increase in Tks- 5 phosphorylation on FAK deletion $\left(n=3,{ }^{* * *} P<0.0001\right)$. (c) ROS production was quantified in B16F10 cells transiently transfected with FAK siRNA and FAK ${ }^{\text {Y397F }}$ using the CM-H2DCF-DA method. Results show an increase in ROS production in cells deficient for FAK, which is not compensated by reexpression of mutant FAK $\left(n=3,{ }^{* \star *} P<0.0001\right)$

and activity remains poorly documented. Nevertheless, as FAK is at the crossroad of FA and actin signaling, it is generally thought to have an important role in the regulation of invadopodia. Here we found that FAK deletion led to an unexpected upregulation of invadopodia activity together with a clear increase in the invasion index in melanoma cells. Moreover, although we show that Src is absolutely required for invadopodia activity, as already reported, ${ }^{35-37}$ FAK has no effect on the activation state of Src in B16F10 cells. Further investigation of potential molecules involved in the control of invadopodia activity revealed that both Tks-5 phosphorylation and ROS production were increased on FAK deletion, suggesting that they may be part of a common signaling pathway in the control of melanoma invasion. This is in agreement with previous result showing that Tks-5 is expressed in human cancer tissues and particularly in breast cancers and melanomas. ${ }^{11}$ Moreover, Tks- 5 has been shown to directly bind to NoxA1, a member of the NADPH oxidase family involved in ROS production, in a Src-dependant manner. ${ }^{38,39}$ In this context, we also show that constitutive activation of Src, via expression of the Src ${ }^{\text {Y530F }}$ mutant, 

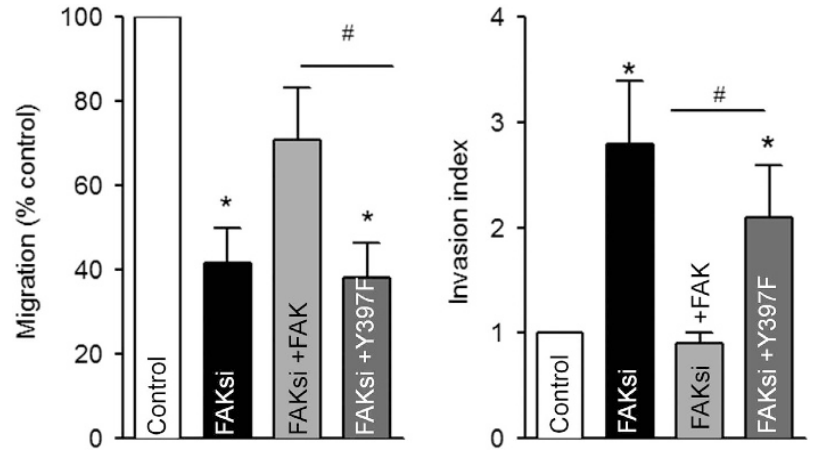

Figure 8 FAK deletion decreases migration but increases invasion. B16F10 cells transiently transfected with control siRNA or FAK siRNA were seeded in transwell inserts. Medium with $10 \%$ FBS was used as a chemotactic agent and the cells were cultured for a further $24 \mathrm{~h}$. For invasion assays, cells were seeded in Matrigel-coated Boyden chambers and allowed to invade and migrate for $24 \mathrm{~h}$. Migrating/invading cell nuclei were stained with DAPI. Note that the decreased migration and increased invasion in FAK-depleted cells partially restored after FAK but not FAK ${ }^{\mathrm{Y} 397 \mathrm{~F}}$ reexpression $\left(n=5,{ }^{*} P<0.05,{ }^{\#} P<0.05\right)$

increased Tks-5 phosphorylation in WT cells but had no additional effect in a FAK-null context, suggesting that in the latter condition, Tks-5 already reached a maximum level of phosphorylation. Taken together, our study suggests that Src promotes Tks-5/ROS-dependent invadopodia activity downstream of FAK in melanoma cells.

This raised the question of how FAK may regulate Src activity. To answer it, we first analyzed the cellular localization of active Src in B16F10 cells. We found that active Src is located at FAs and invadopodia in control cells, whereas in FAK-deficient cells, active Src was almost completely absent at FAs while displaying increased localization at invadopodia. We next analyzed the cellular localization of FAK and found that FAK is located at FAs but not at invadopodia in B16F10 cells. We also show that, in the absence of FAK, melanoma cells display more numerous invadopodia. Conflicting results have been described regarding the cellular localization of FAK at invadopodia. In v-Src-transformed cells, it has been shown that $\mathrm{Src}$ enhanced the interaction between $\beta 1$ integrin and FAK at invadopodia. ${ }^{35}$ Similarly, FAK was also detected in invadopodia from MCF10A-CA1 breast cancer cells where it seems to enhance the activity of these structures. ${ }^{40}$ On the other hand, in the human breast cancer cells, MDA-MB-231 and MTLn3, which display both FA and invadopodia, FAK is not present at invadopodia as well as in rat mammary adenocarcinoma cells where it alters the dynamics of these structures. ${ }^{36,41}$ It is interesting to note that one recent study has mentioned FAK to be dispensable for dot-shaped podosomes formation but essential for the assembly of rosette-shaped structures in Src-transformed fibroblasts and human lung adenocarcinoma. ${ }^{42}$ Finally, in some cancer cell lines, like the HT-1080 fibrosarcoma or the PANC-1 pancreatic cancer cells, FAK has been shown to target MT-MMP1 at FA to mediate matrix degradation. ${ }^{43}$ Therefore, the role of FAK in invasion may depend on the invasive structures being formed by cells originating from different cancer types. Nevertheless, in agreement with previous study, ${ }^{41}$ we found that mutation of FAK at Tyr397, a tyrosine residue that on phosphorylation is a binding site for Src, increases

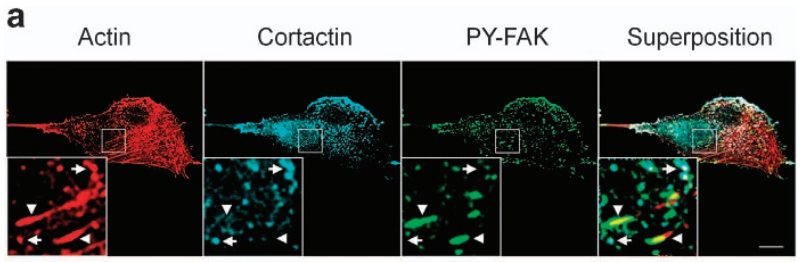

b
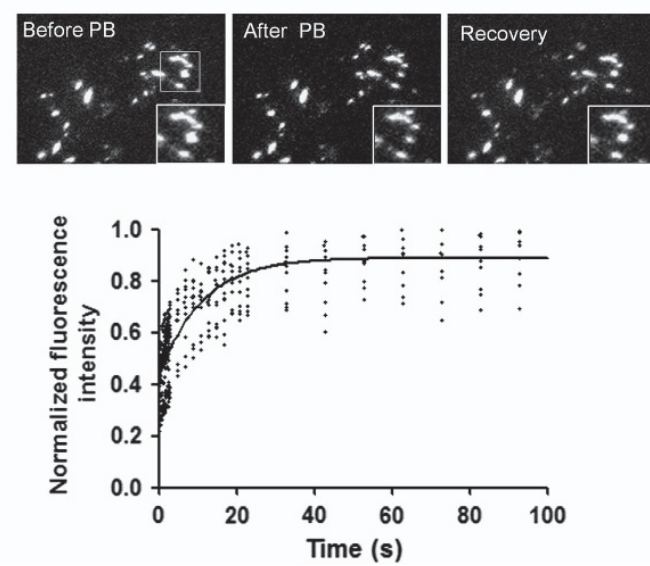

Figure 9 FAK is phosphorylated and highly dynamic at FAs. (a) Confocal images of B16F10 cells immunostained for actin (left panel), cortactin (middle panel) and FAK phosphorylated at Tyr397 (right panel). Note the localization of phosphorylated FAK at FAs (arrowheads) and the colocalization of actin/cortactin at invadopodia (arrows) Scale bars: $20 \mu \mathrm{m}$. (b) B16F10 cells expressing FAK-GFP were imaged by TIRF microscopy before photobleaching (PB) of FA-containing ROls (white squares), immediately after $\mathrm{PB}$ and at the end of the recovery time. Insets show magnified views of the FA being beached. The kinetics of the recovery of FAK-GFP at FA is shown

invadopodia formation and activity in a manner similar to FAK knockdown. This mutated form of FAK also activates Src downstream effectors, Tks- 5 and NoxA, as demonstrated by the increase in Tks-5 phosphorylation and ROS production. Moreover, we show for the first time that FAK deletion in melanoma cells, although decreasing migration, increases the invasion index through Matrigel. These effects were also mimicked by reexpression of FAK ${ }^{\text {Y397F }}$ but not FAK wild type. Thus, our results suggest that, depending on the phosphorylation state of FAK at Tyr397, Src may activate either a migration pathway by triggering FA disassembly via formation of a FAK/Src complex at these sites ${ }^{44-46}$ or an invasion pathway, via specific targeting of Src to invadopodia, leading to matrix degradation. Alternatively, FAK expression may also be environmentally controlled to specify which pathway should be activated (Figure 10).

It has been reported that the lifetime of invadopodia is relatively high, ranging from minutes to hours, ${ }^{31}$ thus allowing significant matrix degradation $3-4 \mathrm{~h}$ after cell plating. Therefore, for efficient ECM proteolysis leading to dissemination of melanoma cells, high migration speed should be either avoided or redirected toward the degradation area. In this respect, our results show that downregulation of FAK is sufficient to both promote increased matrix degradation and reduce migration speed. This leads to the question whether FAK expression could be regulated between different stages of melanoma progression. In their early phase, melanoma cells have been shown to express mainly proliferative genes 
Normal FAK expression
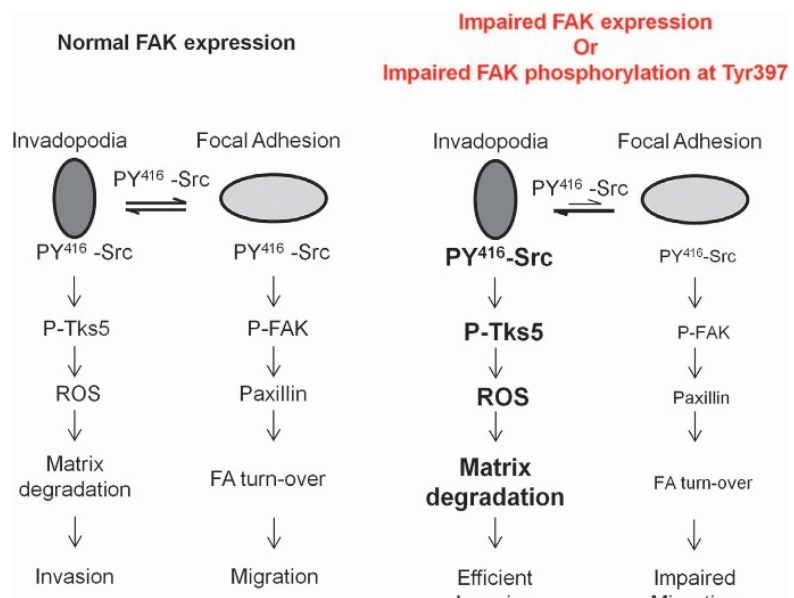

Figure 10 Theoretical model for FAK-mediated regulation of matrix degradation. (left) Active Src is in equilibrium between two compartments: invadopodia and FAs. FAK is located at FAs but not at invadopodia. When phosphorylated at Tyr397, FAK recruits Src to mediate phosphorylation of downstream substrates like paxillin, thus promoting FAs turn-over and cell migration. (right) Depletion of FAK or inhibition of Src binding to FAK releases Src from FAs, thus mediating phosphorylation of Tks-5 at invadopodia to promote invadopodia activity and cell invasion while inhibiting cell migration

which upon unknown signaling change to an invasive signature gene set. ${ }^{47}$ This change may be triggered by subtle alterations of the microenvironment. For example, hypoxia drives metastatic progression by promoting such a switch in melanoma gene signature. ${ }^{48}$ The possible transformation of FAs into invadopodia could represent another example of melanoma plasticity directed toward increased invasion. Indeed, as FA and invadopodia share many structural components, several studies have suggested that invadopodia might derive from FA. Thus, in Src-transformed fibroblasts, invadopodia formation is initiated at adhesions in response to the focal generation of phosphatidylinositol-3,4bisphosphate. Therefore, approaches to identify key molecules that drive the transition between FAs and invadopodia have been used and led to the recent discovery that the combination of increased PI3K with low PKC $\alpha$ activity promotes an invasive state characterized by enhanced formation and activity of invadopodia along with changes in the organization of adhesions. ${ }^{49}$ On the other hand, our FRAP studies revealed that FAK is highly dynamic at melanoma cell adhesions. As FAK is mainly phosphorylated at Y397 at FAs, and therefore targets Src to these sites, a low FAK residence time at FAs would allow high Src availability at invadopodia. Indeed, it is well known that Src trafficking is a fundamental aspect of Src-mediated cell transformation, resulting in Src localization in subcellular structures where both its $\mathrm{SH} 2$ domain and its kinase activity are needed for actin rearrangements. ${ }^{50}$ Moreover, increased stroma rigidity due to fibrosis and ECM remodeling is a hallmark of many cancer cell disseminations. Experimentally, such change in ECM composition could be mimicked, at least in part, by increasing collagen density, which has been recently shown to alter the residence time of $\mathrm{FAK}$, but not that of paxillin, at FA. ${ }^{51}$ Therefore, dynamic regulation of Src/FAK trafficking controlled by the microenvironment may be another means for

melanoma cells to choose between invasion or migration pathways.

In conclusion, our results identify FAK as a key molecule at the crossroad between invasion and migration pathways in melanoma cells. How FAK is environmentally regulated during melanoma progression and whether FAK expression, phosphorylation and/or dynamics represent the key determinants of this transition will need further investigation.

\begin{abstract}
Materials and Methods
Antibodies and other reagents. The following mouse monoclonal antibodies were used: anti-FAK (BD Transduction Laboratories, Le Pont de Claix, France), anti-cortactin (Millipore, Molsheim, France), anti-Tks-5 (Tebu-bio, Le Perray-en-Yvelines, France), anti-Tks-5 PX domain, anti-Tks-5 SH3 domain, antiphosphotyrosine (Millipore), anti-talin, anti-paxillin, anti-phospho-Y397 FAK (Invitrogen, Camarillo, CA, USA) and anti- $\beta$-actin (Sigma-Aldrich, St. Louis, MO, USA). Anti-Src and anti-phospho-Src anti-bodies were from Cell Signalling (Danvers, MA, USA) or Invitrogen (Grand Island, NY, USA). Horseradish peroxidase-conjugated goat anti-mouse was from Promega (Madison, WI, USA). Actin antibody conjugated to Alexa fluor 647 was from Santa Cruz (Heidelberg, Germany). PP2 was from Calbiochem (Billerica, MA, USA) and MnTMPyP from MERCK Biosciences (Darmstadt, Germany). G-sepharose beads and ECL ${ }^{\text {plus }}$ kit were from GE healthcare (Freiburg, Germany). MnPyP, CM-H2DCF-DA, Alexa Fluor 488-conjugated goat anti-mouse, Alexa-405-labelling kit and Lipofectamine 2000 were from Invitrogen. Gelatin from porcine skin and mouse monoclonal Ab was from Sigma-Aldrich.
\end{abstract}

Cell culture, DNA constructs and stable transfection. The B16F10 melanoma cell line was from ATCC (Manassas, VA, USA). Cells were maintained in DMEM (Lonza, Basel, Switzerland) supplemented with 10\% fetal bovine serum, $1 \mathrm{mM} \mathrm{L-glutamine}$ and $1 \%$ penicillin/streptomycin antibiotics. Plasmid pAcGFP1Hyg-C1-FAK was obtained as previously described. ${ }^{52}$ Punctual mutations were performed on the FAK coding sequence through directed mutagenesis (Quickchangell XL site-directed mutagenesis, Agilent Technologies, Massy, France). Y397 was replaced by phenylalanine and the FAK mutation was verified by sequencing. B16F10 cells were transfected with $4 \mu \mathrm{g}$ DNA using lipofectamine 2000 (Invitrogen), according to the manufacturer instructions.

siRNA transfections. B16F10 cells were plated at $1.5 \times 10^{5}$ cells in six-well plates. Cells, $18 \mathrm{~h}$ later, were transfected with $50 \mathrm{pmol}$ of siRNA for $5 \mu \mathrm{l}$ lipofectamine 2000 in $500 \mu$ l Opti-MEM (Gibco, Grand Island, NY, USA). Stealth siRNA oligonucleotides (Invitrogen) used were control siRNA $5^{\prime}$-GAAUCUCAUCU AUUUCGUAACGGAC-3' and FAK SiRNA $5^{\prime}$-UGACAGAUACCUAGCAUCUAGC AAA- $3^{\prime}$. These sequences have already been described to target the $5^{\prime}$-UTR region of FAK in rat MTLn3 cells, ${ }^{41}$ however, a sequence alignment was performed using the link http://www.ba.itb.cnr.it/BIG/Blast/BlastUTR.html to check out sequence homology in Mus musculus as B16F10 cell line stems from murine lineage. Transfected cells were incubated for $48 \mathrm{~h}$ (unless otherwise indicated) at $37^{\circ} \mathrm{C}$ before use.

Cell treatment with PP2, MnTMPyP. One hour after seeding on fluorescent gelatin, B16F10 cells were treated with PP2 [5-(4-chlorophenyl)-7(dimethylethyl)pyrazolo[3,4-d]pyrimidine] for $3 \mathrm{~h}$ at $10 \mu \mathrm{M}$ to inhibit Src activity, and with MnTMPyP [manganese (III) tetrakis(1-methyl-4-pyridyl)porphyrin] for $1 \mathrm{~h}$ at $37^{\circ} \mathrm{C}$ at the concentration of $300 \mu \mathrm{M}$ to inhibit ROS formation.

In vitro fluorescent matrix digestion assay and immunofluorescence. Cells transfected or not with siRNA were plated at $5000 \mathrm{cells} / \mathrm{cm}^{2}$ in a two-well lab-tek and incubated overnight at $37^{\circ} \mathrm{C}$ on fluorescent-labeled Alexa405 gelatin as described elsewhere. ${ }^{53}$ Cells, $18 \mathrm{~h}$ later, were fixed using $4 \%$ paraformaldehyde solution for $15 \mathrm{~min}$, and permeabilized using triton X-100 at $0.05 \%$. Unspecific sites were blocked by incubating cells in $1 \%$ of bovine serum albumin (BSA) at room temperature. Cells were then labeled for $1 \mathrm{~h}$ with anticortactin $A b(1 / 1000)$, anti-talin $A b(1 / 500)$, anti-FAK Ab (1/1000), anti-paxillin Ab $(1 \mu \mathrm{g} / \mathrm{ml})$, anti P-Tyr416-Src Ab (1/250) or Alexa-647-conjugated phalloidin. After three washes with PBS, cells were incubated with Alexa-488-conjugated goat antirabbit $\mathrm{Ab}(1 / 500)$, rhodamine-conjugated donkey anti-mouse $\mathrm{Ab}(1 / 400)$, Alexa- 
555-conjugated goat anti-mouse $\mathrm{Ab}(1 / 250)$ or $\mathrm{CY} 5$-conjugated donkey anti-rabbit $\mathrm{Ab}(1 / 250)$ for $1 \mathrm{~h}$, washed three times with PBS and then observed using either a Leica confocal microscope TSC SPE $(\times 63 \mathrm{HCX}$ PI Apo 1.32 NA objective, Wetzlar, Germany) or a Leica DMIRE2 microscope ( $\times 40$ HCX PI Apo 1.25 NA and $\times 63 \mathrm{HCX}$ PI Apo $1.32 \mathrm{NA}$ objectives). Areas of degradation were identified as black holes on fluorescent gelatin and quantified using ImageJ software downloaded from the $\mathrm{NIH}$ website.

Western blot and immunoprecipitation. Total cell proteins were obtained by lysing cells for $30 \mathrm{~min}$ at $4{ }^{\circ} \mathrm{C}$ in RIPA buffer for western blot $(1 \mathrm{M}$ Tris- $\mathrm{HCl}, \mathrm{pH} 7.5,150 \mathrm{mM} \mathrm{NaCl}, 1 \%$ Triton X-100, $0.1 \%$ SDS, $0.5 \%$ sodium deoxycholate, $2 \mathrm{mM}$ sodium orthovanadate, $10 \mathrm{mM} \mathrm{NaF}, 5 \mathrm{mM}$ Na pyrophosphate) or IP buffer for immunoprecipitation ( $150 \mathrm{mM} \mathrm{NaCl}, 1 \%$ Triton X-100, $1 \%$ Nonidet P-40, $50 \mathrm{mM}$ Tris- $\mathrm{HCl}, \mathrm{pH} 8.0,10 \mathrm{mM} \mathrm{NaF}, 2 \mathrm{mM} \mathrm{Na}_{3} \mathrm{VO}_{4}$ ) both supplemented with Protease Inhibitor Cocktail (complete Mini, Roche, Mannheim, Germany). Lysates were clarified by centrifuging at $14000 \times g$ for $10 \mathrm{~min}$ at $4{ }^{\circ} \mathrm{C}$. Protein concentration was determined using a Biorad kit (Hercules, CA, USA) based on the Lowry colorimetric method. For immunoprecipitation, $500 \mu \mathrm{g}$ of proteins were incubated with precipitating antibody at $1 / 500$ dilution for $3 \mathrm{~h}$ at $4{ }^{\circ} \mathrm{C}$ under rotational shaking following manufacturer instructions. Then, G-sepharose beads (GE Healthcare) were added and incubated a $4^{\circ} \mathrm{C}$. Beads were then washed five times with RIPA buffer, and later suspended in Laemmli buffer. For western blotting, $10 \mu \mathrm{g}$ of proteins were suspended in Leammli buffer and loaded on a $8 \%$ polyacrylamide gel, then transferred to a PVDF membrane. Nonspecific sites were blocked for $1 \mathrm{~h}$ at room temperature using $5 \%$ BSA in TBS-T ((pH 7.4): $10 \mathrm{mM}$ Tris, $150 \mathrm{mM} \mathrm{NaCl}$ and $0.1 \%$ Tween 20). Then, membranes were incubated with primary antibody diluted in TBS-T containing $5 \%$ BSA or $5 \%$ non-fat milk overnight at $4{ }^{\circ} \mathrm{C}$. After washing membranes three times in TBS-T for $10 \mathrm{~min}$, specific horseradish peroxidase-labeled secondary antibodies (Promega) were incubated with membranes for $1 \mathrm{~h}$ at room temperature. Signals were detected using en $\mathrm{ECL}^{\text {plus }}$ kit.

ROS measurement. Cells were plated at subconfluency in complete medium. Cells, $18 \mathrm{~h}$ later, were incubated with $25 \mu \mathrm{M}$ of the fluorescent ROS probe CM-H2-DCF-DA (the 5-(and 6)-carboxy-2', 7'-dichlorodihydrofluorescein diacetate) in HBSS supplemented with $10 \% \mathrm{FBS}$ for $20 \mathrm{~min}$ at $37^{\circ} \mathrm{C}$ in the dark. Cells were then washed carefully three times with HBSS, collected with EDTA for $1 \mathrm{~min}$ and resuspended in HBSS $10 \% \mathrm{FBS}$, then centrifuged for $5 \mathrm{~min}$ at 1000 r.p.m. Cells were subsequently suspended in $300 \mu \mathrm{l} \mathrm{HBSS}$ and analyzed by flow cytometry at $545 \mathrm{~nm}$. Quantification of fluorescence was performed using the FACS Aria software (San Jose, CA, USA).

Cell invasion and migration assay. Invasion was assessed using 24-well plates having Matrigel-coated inserts (BD Biosciences, San Jose, CA, USA) and migration was assessed using uncoated inserts. Transfected B16F10 cells $\left(1 \times 10^{5}\right)$ were seeded on the inner part of the insert $(8 \mu \mathrm{m}$ pore size) in $500 \mu \mathrm{l}$ serum-free DMEM and placed in wells containing $500 \mu \mathrm{l}$ DMEM supplemented with $10 \%$ FBS as chemoattractant. After $24 \mathrm{~h}$ at $37^{\circ} \mathrm{C}$, the inserts' inner surfaces were swabbed to remove noninvasive cells. Cells from the outer side of the membrane were fixed in 4\% paraformaldehyde and stained with 4',6-diamidino2-phenylindole (DAPI). Invading and migrating cells were counted using an epifluorescence microscope. The percent invasion was determined as the ratio of the mean number of cells invading through Matrigel insert membrane over the mean number of cells migrating through non-coated insert membrane. The invasion index is expressed as the ratio of the percent invasion of test cells over the percent invasion of control cells.

Dual FRAP/TIRF experiments. FRAP experiments were done on an iMIC microscope (Till Photonics, Munchen, Germany) equipped with a Cobolt Dual Calypso Laser 491/532 nm (Solna, Sweden) and an Olympus (Rungis, France) $\times 60$ TIRFM (1.45 NA) objective. During acquisition, cells were maintained at $37^{\circ} \mathrm{C}$ in a $5 \% \mathrm{CO}_{2}$ humidified atmosphere using an environmental control system (Life Imaging Services, Basel, Switzerland). Images recorded before and after bleaching were acquired in TIRF mode every $2 \mathrm{~min}$ on an EMCCD camera (Andor Technology, Belfast, Ireland). To avoid possible artifacts of overexpression, only cells expressing low but detectable amounts of protein were chosen for further analysis. Photobleaching was done using a diffraction-limited spot centered at FAs. High-intensity illumination was performed with $50 \%$ of the 491 -nm line for $10 \mathrm{~ms}$. Recovery was followed in TIRF mode at various time intervals until the intensity had reached a steady plateau. For each time point, the intensity of the bleached area was normalized to that of a corresponding unbleached area. Fluorescence during recovery was normalized to the prebleached intensity. FRAP recovery curves were generated using GraphPad Prism 5 (La Jolla, CA, USA).

\section{Conflict of Interest}

The authors declare no conflict of interest.

Acknowledgements. This work was supported in part by grants from the Ligue Contre le Cancer (Comités du Bas-Rhin et du Haut-Rhin) to P.R. K.K.-B. was supported by a doctoral fellowship from the Ministère de la Recherche. R. Vauchelles from the PIQ (Quantitative Imaging Platform, Faculté de Pharmacie, Université de Strasbourg) is thanked for technical assistance in data analyses. We also thank T. Steffan for excellent technical assistance, C. Auger for his help with the ROS measurements and V. Schini-Kerth for providing reagents.

1. Baldassarre M, Ayala I, Beznoussenko G, Giacchetti G, Machesky LM, Luini A et al. Actin dynamics at sites of extracellular matrix degradation. Eur J Cell Biol 2006; 85: 1217-1231.

2. Clark ES, Whigham AS, Yarbrough WG. Weaver AM. Cortactin is an essential regulator of matrix metalloproteinase secretion and extracellular matrix degradation in invadopodia. Cancer Res 2007; 67: 4227-4235.

3. Artym VV, Zhang Y, Seillier-Moiseiwitsch F, Yamada KM, Mueller SC. Dynamic interactions of cortactin and membrane type 1 matrix metalloproteinase at invadopodia: defining the stages of invadopodia formation and function. Cancer Res 2006; 66: 3034-3043.

4. Mueller SC, Chen WT. Cellular invasion into matrix beads: localization of beta 1 integrins and fibronectin to the invadopodia. J Cell Sci 1991; 99(Pt 2): 213-225

5. Hofmann UB, Westphal JR, Waas ET, Zendman AJ, Cornelissen IM, Ruiter DJ et al. Matrix metalloproteinases in human melanoma cell lines and xenografts: increased expression of activated matrix metalloproteinase-2 (MMP-2) correlates with melanoma progression. Br J Cancer 1999; 81: 774-782.

6. Hofmann UB, Westphal JR, Zendman AJ, Becker JC, Ruiter DJ, van Muijen GN. Expression and activation of matrix metalloproteinase-2 (MMP-2) and its co-localization with membrane-type 1 matrix metalloproteinase (MT1-MMP) correlate with melanoma progression. J Pathol 2000; 191: 245-256.

7. Kurschat $P$, Wickenhauser $C$, Groth $W$, Krieg $T$, Mauch $C$. Identification of activated matrix metalloproteinase-2 (MMP-2) as the main gelatinolytic enzyme in malignant melanoma by in situ zymography. J Pathol 2002; 197: 179-187.

8. Linder S, Aepfelbacher M. Podosomes: adhesion hot-spots of invasive cells. Trends Cell Biol 2003; 13: 376-385

9. Buccione R, Orth JD, McNiven MA. Foot and mouth: podosomes, invadopodia and circular dorsal ruffles. Nat Rev Mol Cell Biol 2004; 5: 647-657.

10. Weaver AM. Cortactin in tumor invasiveness. Cancer Lett 2008; 265: 157-166.

11. Courtneidge SA, Azucena EF, Pass I, Seals DF, Tesfay L. The SRC substrate Tks5, podosomes (invadopodia), and cancer cell invasion. Cold Spring Harb Symp Quant Biol 2005; 70: 167-171.

12. Roskoski R Jr. Src protein-tyrosine kinase structure and regulation. Biochem Biophys Res Commun 2004; 324: 1155-1164.

13. Diaz B, Shani G, Pass I, Anderson D, Quintavalle M, Courtneidge SA. Tks5-dependent nox-mediated generation of reactive oxygen species is necessary for invadopodia formation. Sci Signal 2009; 2: ra53.

14. Albiges-Rizo C, Destaing O, Fourcade B, Planus E, Block MR. Actin machinery and mechanosensitivity in invadopodia, podosomes and focal adhesions. J Cell Sci 2009; 122(Pt 17): 3037-3049.

15. Zaidel-Bar R, Itzkovitz S, Ma'ayan A, lyengar R, Geiger B. Functional atlas of the integrin adhesome. Nat Cell Biol 2007; 9: 858-867.

16. Mitra SK, Hanson DA, Schlaepfer DD. Focal adhesion kinase: in command and control of cell motility. Nat Rev Mol Cell Biol 2005; 6: 56-68.

17. Schaller MD, Borgman CA, Cobb BS, Vines RR, Reynolds AB, Parsons JT. pp125FAK a structurally distinctive protein-tyrosine kinase associated with focal adhesions. Proc Natl Acad Sci USA 1992; 89: 5192-5196.

18. McLean GW, Carragher NO, Avizienyte E, Evans J, Brunton VG, Frame MC. The role of focal-adhesion kinase in cancer - a new therapeutic opportunity. Nat Rev Cancer 2005; 5: 505-515.

19. Maung K, Easty DJ, Hill SP, Bennett DC. Requirement for focal adhesion kinase in tumor cell adhesion. Oncogene 1999; 18: 6824-6828.

20. Hess AR, Hendrix MJ. Focal adhesion kinase signaling and the aggressive melanoma phenotype. Cell Cycle 2006; 5: 478-480.

21. Hess AR, Postovit LM, Margaryan NV, Seftor EA, Schneider GB, Seftor RE et al. Focal adhesion kinase promotes the aggressive melanoma phenotype. Cancer Res 2005; 65: 9851-9860

22. Saltel $F$, Chabadel A, Bonnelye E, Jurdic P. Actin cytoskeletal organisation in osteoclasts: model to decipher transmigration and matrix degradation. Eur J Cell Biol 2008; 87: 459-468. 
23. Liu JD, Chen SH, Lin CL, Tsai SH, Liang YC. Inhibition of melanoma growth and metastasis by combination with (-)-epigallocatechin-3-gallate and dacarbazine in mice. J Cell Biochem 2001; 83: 631-642.

24. Hamadi A, Deramaudt TB, Takeda K, Ronde P. Src activation and translocation from focal adhesions to membrane ruffles contribute to formation of new adhesion sites. Cell Mol Life Sci 2009; 66: 324-338.

25. Ahmed S. Nanoscopy of cell architecture: the actin-membrane interface. Bioarchitecture 2011; 1: 32-38.

26. Stylli SS, Stacey TT, Verhagen AM, Xu SS, Pass I, Courtneidge SA et al. Nck adaptor proteins link Tks5 to invadopodia actin regulation and ECM degradation. J Cell Sci 2009; 122(Pt 15): 2727-2740.

27. Seals DF, Azucena EF Jr, Pass I, Tesfay L, Gordon R, Woodrow M et al. The adaptor protein Tks5/Fish is required for podosome formation and function, and for the proteasedriven invasion of cancer cells. Cancer Cell 2005; 7: 155-165.

28. Gray-Schopfer V, Wellbrock C, Marais R. Melanoma biology and new targeted therapy. Nature 2007; 445: 851-857.

29. Miller AJ, Mihm MC Jr. Melanoma. N Engl J Med 2006; 355: 51-65.

30. Destaing O, Block MR, Planus E, Albiges-Rizo C. Invadosome regulation by adhesion signaling. Curr Opin Cell Biol 2011; 23: 597-606.

31. Murphy DA, Courtneidge SA. The 'ins' and 'outs' of podosomes and invadopodia: characteristics, formation and function. Nat Rev Mol Cell Biol 2011; 12: 413-426.

32. Ayala I, Baldassarre M, Giacchetti G, Caldieri G, Tete S, Luini A et al. Multiple regulatory inputs converge on cortactin to control invadopodia biogenesis and extracellular matrix degradation. J Cell Sci 2008; 121(Pt 3): 369-378.

33. Oikawa T, Oyama M, Kozuka-Hata H, Uehara S, Udagawa N, Saya $\mathrm{H}$ et al. Tks5-dependent formation of circumferential podosomes/invadopodia mediates cell-cell fusion. J Cell Biol 2012; 197: 553-568.

34. Schaller MD. Cellular functions of FAK kinases: insight into molecular mechanisms and novel functions. J Cell Sci 2010; 123(Pt 7): 1007-1013.

35. Hauck CR, Hsia DA, llic D, Schlaepfer DD. v-Src SH3-enhanced interaction with focal adhesion kinase at beta 1 integrin-containing invadopodia promotes cell invasion. $J$ Biol Chem 2002; 277: 12487-12490.

36. Bowden ET, Onikoyi E, Slack R, Myoui A, Yoneda T, Yamada KM et al. Co-localization of cortactin and phosphotyrosine identifies active invadopodia in human breast cancer cells. Exp Cell Res 2006; 312: 1240-1253.

37. Bharti S, Inoue H, Bharti K, Hirsch DS, Nie Z, Yoon HY et al. Src-dependent phosphorylation of ASAP1 regulates podosomes. Mol Cell Biol 2007; 27: 8271-8283.

38. Gianni D, Taulet N, DerMardirossian C, Bokoch GM. c-Src-mediated phosphorylation of NoxA1 and Tks4 induces the reactive oxygen species (ROS)-dependent formation of functional invadopodia in human colon cancer cells. Mol Biol Cell 2010; 21: 4287-4298.

39. Gianni D, DerMardirossian C, Bokoch GM. Direct interaction between Tks proteins and the $\mathrm{N}$-terminal proline-rich region (PRR) of NoxA1 mediates Nox1-dependent ROS generation. Eur J Cell Biol 2011; 90: 164-171.

40. Alexander NR, Branch KM, Parekh A, Clark ES, Iwueke IC, Guelcher SA et al. Extracellular matrix rigidity promotes invadopodia activity. Curr Biol 2008; 18: 1295-1299.

41. Chan KT, Cortesio CL, Huttenlocher A. FAK alters invadopodia and focal adhesion composition and dynamics to regulate breast cancer invasion. J Cell Biol 2009; 185 357-370.
42. Pan YR, Chen $\mathrm{CL}$, Chen HC. FAK is required for the assembly of podosome rosettes. J Cell Biol 2011; 195: 113-129.

43. Wang $Y$, McNiven MA. Invasive matrix degradation at focal adhesions occurs via protease recruitment by a FAK-p130Cas complex. J Cell Biol 2012; 196: 375-385.

44. Carragher NO, Westhoff MA, Fincham VJ, Schaller MD, Frame MC. A novel role for FAK as a protease-targeting adaptor protein: regulation by p42 ERK and Src. Curr Biol 2003; 13: 1442-1450.

45. Webb DJ, Donais K, Whitmore LA, Thomas SM, Turner CE, Parsons JT et al. FAK-Src signalling through paxillin, ERK and MLCK regulates adhesion disassembly. Nat Cell Biol 2004; 6: 154-161.

46. Deramaudt TB, Dujardin D, Hamadi A, Noulet F, Kolli K, De Mey J et al. FAK phosphorylation at Tyr-925 regulates cross-talk between focal adhesion turnover and cell protrusion. Mol Biol Cell 2011; 22: 964-975.

47. Hoek KS, Eichhoff OM, Schlegel NC, Dobbeling U, Kobert N, Schaerer $L$ et al. In vivo switching of human melanoma cells between proliferative and invasive states. Cancer Res 2008; 68: 650-656.

48. Widmer DS, Hoek KS, Cheng PF, Eichhoff OM, Biedermann T, Raaijmakers Ml et al. Hypoxia contributes to melanoma heterogeneity by triggering HIF1alpha-dependent phenotype switching. J Invest Dermatol 2013; 133: 2436-2443.

49. Hoshino D, Jourquin J, Emmons SW, Miller T, Goldgof M, Costello K et al. Network analysis of the focal adhesion to invadopodia transition identifies a PI3K-PKCalpha invasive signaling axis. Sci Signal 2012; 5: ra66.

50. Timpson P, Jones GE, Frame MC, Brunton VG. Coordination of cell polarization and migration by the Rho family GTPases requires Src tyrosine kinase activity. Curr Biol 2001; 11: $1836-1846$

51. Le Devedec SE, Geverts B, e Bont H, Yan K, Verbeek FJ, Houtsmuller AB et al. The residence time of focal adhesion kinase (FAK) and paxillin at focal adhesions in renal epithelial cells is determined by adhesion size, strength and life cycle status. J Cell Sci 2012; 125(Pt 19): 4498-4506.

52. Deramaudt TB, Dujardin D, Noulet F, Martin S, Vauchelles R, Takeda K et al. Altering FAKpaxillin interactions reduces adhesion, migration and invasion processes. PLOS One 2014; 9: e92059.

53. Artym VV, Yamada KM, Mueller SC. ECM degradation assays for analyzing local cell invasion. Methods Mol Biol 2009; 522: 211-219.

cc) (i) $\ominus$ Cell Death and Disease is an open-access journal (c) ${ }_{\mathrm{BC}} \mathrm{ND}$ published by Nature Publishing Group. This work is licensed under a Creative Commons Attribution-NonCommercialNoDerivs 3.0 Unported License. The images or other third party material in this article are included in the article's Creative Commons license, unless indicated otherwise in the credit line; if the material is not included under the Creative Commons license, users will need to obtain permission from the license holder to reproduce the material. To view a copy of this license, visit http://creativecommons.org/licenses/ by-nc-nd/3.0/

Supplementary Information accompanies this paper on Cell Death and Disease website (http://www.nature.com/cddis) 